\title{
Phase III Trial
}

National Cancer Institute

\section{Source}

National Cancer Institute. Phase III Trial. NCI Thesaurus. Code C15602.

A clinical research protocol designed to investigate the efficacy of the biomedical or behavioral intervention in large groups of human subjects (from several hundred to several thousand), to confirm efficacy, to monitor adverse reactions to the new medication or treatment regimen with respect to long-term use and by comparing the intervention to other standard or experimental interventions as well as to a placebo. 